Chapter 6

\title{
Like a Canary in the Coal Mine: Behavioral Change as an Early Warning Sign of Neurotoxicological Damage
}

\author{
Kathleen M. Raley-Susman \\ Additional information is available at the end of the chapter \\ http://dx.doi.org/10.5772/57170
}

\section{Introduction}

With more than 7 billion people in the world, global food production is of critical importance. Back in the 1960's, when population models predicted such a large human population, agricultural scientists and policy makers felt an urgent need to dramatically increase global food production and to radically change how food was grown and distributed. Science, business and governments collaborated to engineer a paradigm shift in how we feed our species. Galvanized by the efforts of Norman Borlaug to successfully develop high-yield and disease-resistant wheat, the Green Revolution led to an order of magnitude increase in global food production in fewer than forty years [1]. Both the Rockefeller and Ford Foundations worked with international governments to use a combination of agricultural and technological approaches to vastly increase the productivity of the major cereal crops that feed the world: wheat, corn, rice, and later other important crops.

The major changes in agricultural practice included the selective breeding of high yield crop varieties, changes to irrigation approaches, increased mechanization of farms and the use of fertilizers and pesticides. The high-yield varieties were selectively bred to produce large and many seeds and to have shorter and sturdier stems to support the seeds. They matured quickly and were much less sensitive to photoperiod, enabling a longer growing season or multiple growing seasons in a year. They were also bred to respond to externally applied fertilizers with fast growth and rapid maturation [1]. Farmers were eager to plant the super-crops and soon changed agricultural approaches to focus exclusively on a few or one variety, giving rise to the monoculture agriculture and large mechanized farming that dominates global agricultural practice today, particularly in the developed world. These varieties are dependent on plentiful irrigation and fertilizer application. To protect the new crops and further enhance yield, farmers applied a growing arsenal of chemical pesticides, some of them derived from chemicals produced in World War I and World War II as chemical warfare agents. 
The Green Revolution prevented countless deaths due to starvation and created jobs and entire new industries. Huge mega-companies devoted to the production of pesticides, fertilizers and seeds now dominate big agri-business. More than one third of the world's workers are employed in agriculture. The costs are also enormous. Industrial agriculture has caused widespread damage to the land, water and air. Fertilizer run-off has damaged aquatic ecosystems; soils overplanted with monoculture crops are depleted of nutrients; soil, water and the air are contaminated with a complex mixture of pesticides and other toxic chemicals. What are the consequences of chronic exposure to low levels of mixtures of pesticides in the water we drink, in the air we breathe? How are other organisms affected? How are beneficial soil organisms influenced? There is growing awareness of the human health and environmental health consequences of our high-yield agricultural practices. An increasing number of studies indicate that most animals are affected deleteriously by the pervasive presence of pesticides in the environment, humans included.

This chapter focuses on the effects of pesticides and pesticide mixtures on unintended organisms. I explore the behavioral consequences of exposure to toxic chemicals in the environment, with a focus on the use of behavioral change as an early marker of potential or ongoing damage to the nervous system. Recent work in model organisms underscores the value of behavioral assessment of toxicity. Because of the substantial evolutionary conservation of the molecular targets of many pesticide chemicals, what we learn about in non-human animals can readily be applied to human health. We are all in this together.

\section{The most common pesticides}

The hundreds of pesticides in existence fall into five main categories: organophosphates, organochlorines, carbamates, glyphosates and neonicotinoids. Agricultural workers are often exposed to high doses, particularly over their lifetime. In addition, most rivers and lakes, nonagricultural soil and even the air contain measurable, albeit lower, concentrations of all these chemicals. The vast majority of pesticides in use to protect plants from herbivory target the control of muscle contraction, leading to paralysis and death of the intended target insects and other arthropods. Fungicides and herbicides target enzyme pathways that result in the death of harmful fungi and weeds, but the chemicals can have unwitting effects on animals as well. In order to better understand how these pesticides operate in animals, I will first briefly review the mechanism by which muscles are activated by nerves.

\subsection{A Key target of pesticides: The neuromuscular junction}

The connection between motor neurons that generate movement and the skeletal muscles they activate is called the neuromuscular junction (NMJ). This synapse is highly specialized to afford rapid and reliable activation of the muscle. In vertebrates, all skeletal muscles are activated by one type of neuron, the motor neuron, which uses the neurotransmitter acetylcholine (Figure 1). The presynaptic terminal of the neuromuscular junction contains hundreds of synaptic vesicles, each loaded with 5000-10,000 molecules of acetylcholine. A single action 
potential firing from one motor neuron can cause the release of as many as 300 vesicles within a millisecond or so [2]. The 1.5 million molecules of transmitter rapidly diffuse across the cleft between the nerve and muscle cell and interact with 1000-2000 receptor channels that are densely clustered at the synaptic junction membrane [3], leading to a large depolarization that initiates a muscle contraction. The cytoarchitecture of this synaptic connection has a tremendously large safety factor, to ensure a 1:1 correspondence between an action potential in the nerve and a corresponding action potential (leading to a contraction cycle) in the muscle cell.

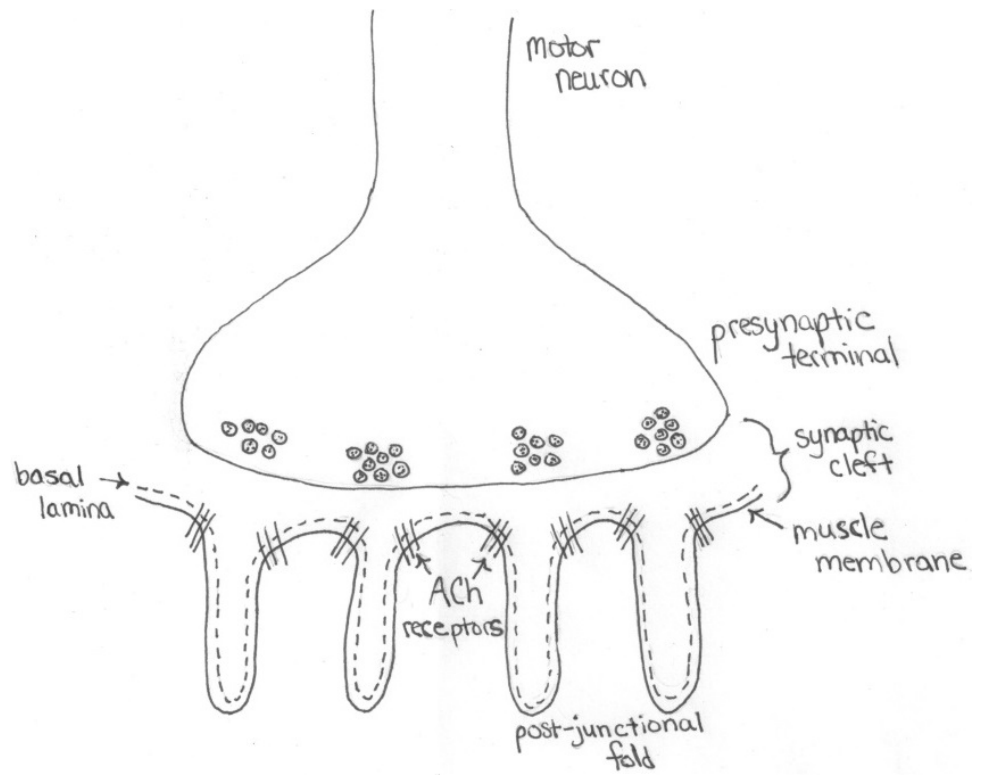

Figure 1. Vertebrate Neuromuscular Junction

At the vertebrate neuromuscular junction, the presynaptic nerve membrane and the postsynaptic muscle membrane are separated by a small space called the synaptic cleft that also contains an extracellular matrix-derived basal lamina that adheres tightly to the muscle membrane (Figure 1). Composed of structural molecules like collagen type -IV and laminins [4], the basal lamina also contains a form of acetylcholinesterase with a collagen tail (ColQAChE). The ColQ tail localizes this form of acetylcholinesterase to the NMJ [5]. Acetylcholinesterase is the product of a single, highly conserved vertebrate gene, and many different versions are generated by alternative splicing, co-translational and post-translational modifications and even the formation of oligomers [6], differing in tissue expression and subcellular localization. The catalytic region of the enzyme is very highly conserved across organisms. As a consequence, chemicals that generally affect cholinesterases can have effects on multiple tissues in multiple organisms. 
There are hundreds of different proteins involved in the molecular mechanism of neurotransmitter release and the majority of these proteins are involved in the release of all neurotransmitters. The proteins govern the movement of synaptic vesicles to the presynaptic terminal membrane, the fusion of the vesicles with the membrane so as to release the chemical neurotransmitter, the recycling of the vesicle membranes back into the terminal and the packaging of neurotransmitter molecules back into vesicles. These important molecular players are highly conserved evolutionarily, being present in mammals, amphibians, flies and nematodes. In addition, all vertebrates utilize the same overall scheme for muscle contraction. Many of these proteins are found in all animals, including invertebrates. Nonetheless, there can be substantial genetic variation in the proteins, with differences in function and structure in vertebrate as compared with invertebrate neurotransmission proteins. The evolutionary divergence of these proteins is important in the design of pesticides that are selective for invertebrate target species, while at the same time being relatively less toxic to non-target animals. However, many of the pesticides currently in use do have considerable cross-reactivity with non-target organisms, depending on the dose and exposure methods used.

Invertebrates have an overall similar mechanism by which motor neurons excite muscles and many of the same proteins are critical for the release of neurotransmitter. There are a number of differences, however. While acetylcholine is the major excitatory neurotransmitter used by motor neurons in a large number of invertebrates including nematodes, annelids, arachnids and mollusks, insects and other arthropods utilize glutamate as a major excitatory neurotransmitter at the neuromuscular junction, with glutamate acting on postsynaptic muscle membrane glutamatergic sodium channels. Another key difference is that many invertebrates also control muscle movement via inhibitory motor neurons that utilize GABA or glycine as a neurotransmitter. These inhibitory motor neurons synapse directly on muscle fibers [7]. This local control of muscle function is quite different from the central spinal cord-level control in vertebrates [8]. Another distinction between vertebrate and invertebrate muscle control is that arthropods in particular modulate muscle function peripherally, rather than at the level of the central nervous system, via a wide variety of circulating hormones and neuromodulators [7]. Many invertebrate motor neurons release more than one neurotransmitter, achieving modulation of muscle force directly at the neuromuscular junction [7]. While ACh is an important neurotransmitter across all animals, the principle effect of pesticides directed at the acetylcholine neurotransmitter system is to alter central nervous function in arthropods.

\subsection{Organophosphates}

More than half of the insecticides in use across the globe fall into the organophosphorous group of chemicals. The major organophosphates in use today are chlorpyrifos, parathion, malathion and diazinon. Chlorpyrifos is the major compound used in the United States and Europe. Organophosphates were first developed as nerve gases in the 1930's in Nazi Germany [9]. After World War II, American chemists developed this class of insecticide based on those materials. It is estimated that more than a billion pounds are produced worldwide each year [9].

Organophosphates target the enzyme acetylcholinesterase, which is a member of the serine hydrolase enzyme superfamily [10]. This enzyme plays a critical role in nerve and muscle 
activity in vertebrates and some invertebrates (Fig. 1), and in central nervous system function in many animals, including arthropods. The organic groups of organophosphate pesticides can be modified to affect target specificity, penetration of the chemical internally, water solubility and persistence in the environment. All of the major organophosphorous pesticides in use for agricultural and residence purposes have a common mechanism of action, that of phosphorylating acetylcholinesterase, thereby inactivating it [11]. This covalent modification is permanent. The inhibition of acetylcholinesterase activity, particularly at the neuromuscular junction, reduces the clearance of acetylcholine from the synaptic, resulting in prolonged neuromuscular stimulation, which causes seizures and paralysis. In arthropods, the effect is primarily within central regulatory neurons and sensory systems [12].

\subsubsection{Unintended targets of organophosphorus pesticides}

When a pesticide is developed for use, it must be approved by governmental regulatory agencies in many countries. The Environmental Protection Agency (EPA) in the United States requires testing of toxicity to a variety of animals, usually assessing lethality at working doses recommended by the pesticide-producing company, as well as assessing potential cancer risk. The EPA requires pesticides be assessed for ecological as well as human health risks. A company seeking to gain EPA approval to sell and distribute a pesticide conducts the scientific tests and files the regulatory materials. Pesticides that have gained approval are periodically reassessed based on reports received from scientific study of risk and reports of problems submitted to the EPA. Ecological assessment data include toxicological studies on wildlife and plants that represent non-target organisms likely to be exposed unintentionally through runoff, aerial drift or bioaccumulation. For this testing, organisms are exposed to different concentrations of the "active ingredient" of the pesticide being assessed, usually in isolation, without other so-called inactive ingredients. Both short and long-term effects of the active ingredient are measured including lethality, growth and reproduction rates. In addition, residue measurements are conducted to determine degradation of the pesticide, possible toxicity of breakdown metabolites, persistence and ability to travel in the environment (soil, water, air, bioaccumulation). If the regulatory commission (like the EPA) believes that a particular type of ecosystem is not likely to be exposed to a candidate pesticide, then the registration materials stipulate that the pesticide is either "safe" or poses no "reasonable harm" to the organisms within that ecosystem, even if direct evaluation of risk or exposure has not been conducted. If the assessors predict a high likelihood a particular ecosystem will be exposed, then additional tests are often required [13].

Acetylcholinesterase, the major target enzyme for organophosphorus pesticides, is a member of a very large class of carboxylic esterases [14]. Most organophosphates interact with a serine group present at the active site of the enzyme. However, they can also affect other enzymes, including other serine hydrolases and even serine proteases [15]. As a result, many cell processes and cell signaling pathways can be disrupted, including serotonin and dopamine neurotransmission, growth regulation, hormonal regulation and other systems. Of particular concern to epidemiologists are the possible long-term consequences of exposure during key developmental critical periods. For example, $83 \%$ of pregnant women in the U.S. had detect- 
able levels of an organophosphate metabolite in their urine [16]. At the same time, a $600 \%$ increase in autism incidence has been reported in California [17]. Mothers living in the California Central Valley who had been exposed to pesticides while pregnant gave birth to children who were 7.6 times more likely to be diagnosed with Autism Spectrum Disorder [18] and there was a $230 \%$ increase in maternally reported pervasive developmental disorders in children whose mothers had measurable organophosphate metabolites in their urine [19]. Cholinergic abnormalities have been reported in autism [20]. While not conclusive, studies such as these and others [20] indicate that gestational exposure to pesticides might well influence neural development. Direct experimental animal studies support these concerns. Exposure to chlorpyrifos during the early postnatal period altered rat memory function and spatial navigation in adulthood [21]. Chick embryos exposed to low levels of chlorpyrifos showed reduced head development [22] and axon development was disrupted in exposed zebra fish embryos [23].

Acetylcholinesterase (AChE) inhibition, affecting neural cholinergic signaling during development, is a likely cause of organophosphorus pesticide effects on behavior, growth, and reproduction. However, most organophosphorus pesticides can also affect other enzyme systems that involve serine esterases, serine hydrolases or serine proteases. This large and essential group of enzymes is important for overall cell and body metabolism, immune and endocrine system functioning, in addition to nervous system functioning. These enzymes differ in their sensitivities to different organophosphorus pesticides, from essentially insensitive to highly sensitive to inhibition. Doses of organophosphate compounds that exquisitely affect AChE activity might have little or no effect on other members of this enzyme superfamily. However, many unintended target enzymes are affected by concentrations of organophosphate compounds or their metabolites that have little effect on AChE. For example, several organophosphates that induce a delayed neuropathy are relatively less reactive with AChE [24]. Further, metabolites of organophosphorus insecticides can also exert effects by reacting with non-target members of the serine hydrolase enzyme superfamily [25]. Indeed, such interactions with serine hydrolases in liver are important means for detoxification [25]. Recent work on the environmentally relevant metabolites of chlorpyrifos and parathion demonstrated substantial inhibition of the activity of key liver carboxylesterases [25]. Several lipases important in brain function are sensitive to organophosphates. Neuropathy target esterase (NTE) is one such enzyme, first discovered as the target enzyme associated with a lethal neuropathy caused by a ginger extract substitute added to drinks during Prohibition in the 1930's [10], an insecticide that was also used as a lubricant for machine parts.

Over the past 15 years or so since the introduction of organophosphorus pesticides as a safer alternative to DDT and other organochlorine pesticides, increasing evidence indicates that organophosphates are harmful to immune function through both acetylcholinesterase inhibition and via noncholinergic mechanisms. Vertebrates and invertebrates share common cell types and mechanisms of innate immunity, namely the presence of phagocytic cells (monocytes and neutrophils), the production of cytokines [26] and a histocompatibility cell recognition system. The complex adaptive immune responses, in particular T- and B-lymphocyte antibody-mediated immune systems first appeared with the evolution of the vertebrates. 
Serine hydrolases play important roles in innate immune function and could be unintended targets of organophosphates [27]. Malathion, at doses that inhibit acetylcholinesterase, suppressed humoral immune responses in mice exposed under laboratory conditions [28] and inhibited cytotoxic T-lymphocyte responses [29]. At low doses, below those that inhibit AChE, malathion stimulated immune activation in rodents [30]. Immunotoxic effects have also been reported in birds, fish, small mammals and soil invertebrates exposed to chlorpyrifos, malathion and diazinon [26]. Parathion, diazinon and chlorpyrifos have also each been shown to alter immune function [26]. Reports on earthworms have shown reduced immune function after exposure to organophosphorus compounds [31, 32]. Laboratory studies in fish also demonstrated an immunosuppression after sublethal organophosphate exposure to malathion [33] and diazinon [34]. A number of transcription factors important for cell division and differentiation are inhibited by chlorpyrifos [35]. These studies are complex and can suffer from interpretational difficulties and clearly much more work is needed. Nonetheless, the increasing reports of unintended effects on many different organ systems are cause for concern.

\subsubsection{Organophosphorus pesticides act as endocrine disrupters (EDC's)}

An endocrine disrupting chemical (EDC) is any chemical that alters an endocrine-regulated system or behavior. Early work centered on chemicals whose structural characteristics allowed them to be estrogen-mimics or estrogen receptor agonists or antagonists. However, more recent work has acknowledged that many chemicals, while not directly interacting with estrogen receptors, can alter endocrine-related functions and so produce long-term effects on growth, metabolism, reproduction and behavior [36].

Developmental exposure to organophosphorus pesticides has widespread effects on neural development, which influences behavior and physiology well into adulthood. Late pre-natal and early postnatal exposure of rats to low concentrations of chlorpyrifos caused impairments in synaptic function, with subsequent locomotory and cognitive dysfunction ([37- 39]). Exposure to very low levels of chlorpyrifos early in gestation, at the time of neurulation, a very early stage of brain development, resulted in locomotory and cognitive abnormalities that persisted into adulthood [40]. The impairments were not severe, suggesting some neurodevelopmental compensation or recruitment of alternate mechanisms to preserve functionality. Indeed, early developmental exposures to chlorpyrifos, parathion, malathion or diazinon have each been associated with alterations in serotonergic and dopaminergic systems [41]. The consequences of alterations in neurotransmitter systems during development, referred to as organizational effects, would have an impact on neuroendocrine system development, thus altering reproductive behavior, in addition to myriad effects on sensory function, cognitive function, predator/prey avoidance and other behaviors. For example, salmon exposed to diazinon were less responsive to predator cues [42]. Organophosphate exposure in development has also been linked to modified oxytocin and arginine vasopressin (AVP) activity [43], altered social behavior [44] and sex-specific behaviors [45].

There is sufficient evidence that developmental exposure to organophosphorus pesticides can cause permanent neurobehavioral and neurological impairments that chlorpyrifos has been banned in the US for residential use. Despite this compelling evidence, however, this and other 
organophosphorus pesticides are still heavily used in agricultural settings, exposing workers, nearby communities and the surrounding aquatic and terrestrial wildlife to these harmful and environmentally persistent chemicals. It is abundantly clear that, while recommended application doses of particular organophosphorus pesticides may not cause overt death, persistent exposure to these compounds, present at biologically active concentrations in water, soil and the food supply, can lead to neurobehavioral changes that influence wildlife and humans.

\subsection{Organochlorine pesticides}

Organochlorine pesticides are among the most environmentally persistent human pollutants, existing in soil, water and air for decades. Developed in 1946, the first commercial herbicides, including 2,4 D and 2,4,5, T were introduced to control broad-leaf weeds. 2,4,5 T was used extensively during the Vietnam war to clear jungle hiding places. Byproducts of that chemical production included dioxin and DDT, which are potent neurotoxins. In the 1940's, DDT was heralded as a potential eradicator of malaria and was sprayed widely as a residential and agricultural insecticide until alarming reports of bird and human morbidity and death in the 1960's. Other banned organochlorine pesticides include aldrin, dieldrin, chlordane and heptachlor. Despite recent bans on their use in some countries, measurable levels of many of these chemicals are still present, even in areas like the Arctic, where they were never used [46].

Others remain in use in the US and in the developing world, including lindane, endosulfan, dicofol, methoxychlor and pentachlorophenol. These chlorinated hydrocarbons are readily lipid soluble, which leads to bioaccumulation in wildlife and in the food chain. Measurable organochlorine residues are found in blood, adipose and breastmilk in humans. Most organochlorines interfere with ion channel function, predominantly in the nervous system. For example, endosulfan binds to and blocks the chloride channel portion of the GABA-a receptor, acting as a noncompetitive antagonist [47]. Lindane, an active ingredient in head lice treatments for children, also interacts with the GABA-a receptor, whereas methoxychlor, chemically similar to DDT, interacts with insect sodium channels, leading to their persistent activation [48]. Lindane and several other organochlorines have been banned from use in many countries because of unintended endocrine-disrupting effects on wildlife and humans (see below) but others, including endosulfan, continue to be used heavily in many parts of the world.

The primary target of endosulfan as an insecticide is to alter ion flux of sodium and potassium, thereby interfering with neuron activity and thus motor function in insects. However, it is a relatively nonspecific insecticide, which is in part why it is used widely for pest protection of many important crops worldwide, as well as for a preservation treatment for wood. Endosulfan, known to be toxic to fish and other aquatic organisms [47], blocks the GABA receptor ion channels, interfering with critical inhibitory neuronal function in vertebrates, resulting in hyperexcitability of many neuronal circuits. Endosulfan is highly toxic to fish and aquatic invertebrates [49] and has also been found to be genotoxic and neurotoxic to mammals [50]. Endosulfan can be transported by air and water and measurable and increasing levels have been reported in the air over the Arctic [50] and even within tissues of animals living large 
distances from regions that use pesticides, like polar bears in the Arctic [46]. As a result, endosulfan used in one nation will affect many other nations that might have banned its use. Organochlorines are found globally and in every ecosystem that has been studied [36].

\subsubsection{Unintended targets of organochlorines}

Organochlorines, like organophosphates, have a number of unintended effects, the nature of which varies with exposure dose/time, species, developmental stage and method of exposure. Chronic sublethal exposure of adult rats to endosulfan in food led to hair loss, enlarged kidneys and increased liver toxicity. Higher doses led to increased incidence of aneurysms [47]. Dogs exposed to endosulfan exhibited increased sensitivity to noise, jerky or tonic movements, excessive abdominal contractions and neurotoxicity [47]. These effects are not related to their intended effects on GABA-a channels and occur at lower concentrations.

In addition to the acute and pervasive neurotoxicity of the major organochlorine pesticides, the compounds in lower concentrations act as endocrine disrupting chemicals (EDC's). Low levels of endosulfan impaired sexual pheromone communication and mating success in newts (Notophthalmus viridescens) [51] and methoxychlor affected scent-marking behavior in mice [52]. Further, mice exposed to methoxychlor in utero exhibited altered exploratory behavior when adults [53]. Frogs exposed to endosulfan exhibited convulsions, followed by temporary paralysis [54]. Rats exposed to endosulfan as adults or early in development exhibit an array of neurological alterations, including impairments in learning and memory, increased spontaneous motor activity, reduced escape and avoidance behavior learning and increased fighting behavior [47]. Hormones modulate these behaviors, particularly during neural development. Thus, while endosulfan is not a direct endocrine disrupter [47], its potent effects on neural systems may indirectly influence many endocrine-regulated functions.

\subsection{Carbamates}

Carbamates, introduced in the 1950's, are organic insecticides and fungicides that were originally derived from the extracts of the West African calabar bean. This class of pesticide inhibits acetylcholinesterase activity much like organophosphate pesticides, but the effects are reversible. Carbamates degrade fairly rapidly in water, with half-lives ranging from 1 day to 4 weeks. The liver detoxifies carbamates and the metabolites are excreted via the kidneys. The major insecticides are aldicarb, carbofuran (Furadan) and carbaryl (Sevin). Carbamates are also found in cosmetics, as preservatives and in polyurethanes, so their presence in the home is quite pervasive [55]. Humans are exposed via skin absorption, ingestion and inhalation and acute toxicity symptoms include headache, nausea, and dizziness. Carbamates are the class of insecticides most prevalent for in-home use. Dithiocarbamates, in contrast, while still rapidly degraded, have little to no effect on acetylcholinesterase activity but rather bind divalent metal ions like manganese and zinc and are used as potent fungicides for home and agricultural use.

Carbamates, despite their rapid degradation and overall low persistence and mobility within the environment, are highly toxic to a wide range of vertebrates and invertebrates. Insecticidal carbamates cause the same symptoms of neurotoxicity as the organophosphate pesticides, with 
a similar wide range of LD50 values based on chemical modifications [55]. Because the chemicals have low persistence and low mobility, and because the actions are reversible, carbamates are generally considered safe for humans and wildlife, except for occupational or intentional exposure to high concentrations. Very little work on possible long-term consequences of brief or transient exposure to the chemicals has been done, nor has there been much work on possible genotoxic, carcinogenic or reproductive effects. A recent review of the available epidemiological studies of carbamates indicated variable cancer risk from occupational exposure to different carbamates [56]. A few reports indicate reproductive and developmental problems in children of exposed workers [57, 58], suggesting possible transgenerational effects.

There is growing concern about carbamates, particularly thiocarbamates, and in particular the associated metal toxicity. A recent human epidemiological study implicated the carbamate fungicide ziram in the etiology of Parkinson's disease [59]. The risk of Parkinson's disease was significantly higher when patients were exposed to the combination of ziram, maneb (another carbamate fungicide) and paraquat, for patients exposed in both occupational and residential areas, and for patients exposed at a younger age [59]. The manganese associated with several common dithiocarbamate fungicides is released when the organic carbamate moiety is degraded or metabolized which can enhance the toxicity of these compounds [60]. Manganese is a potent neurotoxin that is particularly harmful to dopamine neurons [61], causing toxicity via oxidative stress, mitochondrial inhibition and the production of reactive oxygen radicals. Dopamine neurons are particularly vulnerable because both extracellular and intracellular dopamine is enzymatically oxidized to a reactive species in the presence of manganese [61]. Mancozeb exposure, while not lethal to nematodes, inhibited larval growth, induced a heat shock response and altered gene expression [60]. These results suggest that, while dithiocarbamate fungicide might not be lethal or toxic in the short-term, it might cause long-term changes in neuron function that influence behavior and health later in life.

\subsection{Glyphosate (Roundup)}

Introduced by Monsanto in the 1970's, glyphosate is one of the most prevalent herbicides in use today. In 2007, the US applied 185 million pounds of Roundup for agricultural use alone [62]. Monsanto also developed glyphosate-resistant crops, so many farmers plant these crops and apply copious amounts of Roundup. It is also the herbicide most used residentially and on corporate campuses for lawn and garden maintenance.

The primary mechanism of action of glyphosate is to inhibit the enzyme 5-enolpyruvylshikimic acid-3- phosphate synthase, an enzyme found only in plants and microorganisms that is responsible for the synthesis of tyrosine, tryptophan and phenylalanine [63]. Because animals obtain these amino acids from dietary sources, glyphosate has been heralded as a safe and effective herbicide for general use. Glyphosate is considered non-carcinogenic and of very low toxicity to humans. It is less persistent in water than in soil, where it can be retained for over a year. Glyphosate has been considered only slightly toxic to amphibians and fish because of its 12-60 day persistence in water [64]. 
With its widespread use, glyphosate levels are measurable in most soil, water and even air. Low levels have been shown to inhibit steroidogenesis [65] and to alter testosterone levels and testicular morphology in rats exposed pre-pubescently to glyphosate [66]. There are also reports that the commercial formulations of Roundup, which contain inactive ingredients like surfactants to enhance persistence and ease of application, have teratogenic effects in amphibians and other vertebrates $[67,68]$. Several studies have demonstrated that Roundup inhibits aromatase, which can have profound neuroendocrine effects [69], although these findings are controversial because these effects have so far only been demonstrated in isolated human cells. A recent report provided evidence that male offspring of female rats exposed to concentrations of Roundup in their drinking water that are found in the environment exhibited alterations in genes important for thyroid function [66]. These reports, taken together, suggest that glyphosate, or the combination of glyphosate and its inactive ingredients in commercial formulations, may cause changes in neural and neuroendocrine function that could have long-term consequences.

\subsection{Neonicotinoids}

In the 1980's and 1990's, largely in response to the toxicity caused by organophosphate and organochlorine pesticides, a new class of insecticide was developed based on the naturally occurring plant alkaloid nicotine, which is known to have a higher toxicity in insects than in mammals. Bayer Corporation introduced imidacloprid in the 1990's and it is now the most widely used neonicotinoid insecticide in the world [70]. Imidacloprid and two other prevalent neonicotinoids, clothianidin and thiamethoxam, are nicotine-related compounds that are photostable, water-soluble and environmentally persistent. Because they act selectively on the acetylcholine receptor, much like nicotine (Figure 1), it is thought that there are far fewer unintended targets for the compounds. Neonicotinoids are not metabolized by acetylcholinesterase and their clearance from tissue is slow and so their action on the acetylcholine receptor is essentially irreversible. The neonicotinoid insecticides have a much higher affinity for the insect nicotinic acetylcholine receptor than for other such receptors, leading to a lower toxicity in non-insect animals [71].

Neonicotinoids are of deep concern because they act with high potency against target insect pests as well as beneficial insects like honey bees and other important pollinators. A host of recent work has indicated that low-level exposures, of the magnitude measured in agricultural dust drift, pollen and nectar, impairs honey bee navigation, foraging and queen production [72], particularly when exposures occur alongside other pesticides [73].

Even though neonicotinoids are not lethal to non-insects, low, sublethal exposures impaired feeding behavior in a crucial benthic freshwater invertebrate, Gammarus pulex [74], and reduced exploratory and burrowing behavior in two different species of earthworm [75]. Further, male offspring of female mice exposed to chothianidin during gestation and lactation had long-term alterations in exploratory behavior and increased spontaneous locomotory activity [76]. These findings suggest that neonicotinoids are not safe for non-target organisms. 


\section{Assessing behavioral change rather than lethality}

Determining whether a pesticide is harmful to humans or wildlife is a tricky business. Many different factors are important. How the chemical spreads through water, soil, and air affects the extent of pesticide exposure. How the chemical is applied (spraying, irrigation water, seed coating) also affects how it spreads through the environment. Temperature, $\mathrm{pH}$, sunlight and other environmental conditions affect the persistence or breakdown of the chemical. The biological activity of the active ingredient can be altered by other so-called inert ingredients contained within the commercial formulation, like surfactants or solubilizers. The breakdown products might also carry risks in and of themselves. The action of a particular compound might also be different if it is present in a mixture of other pesticides. The concentration of the compound can also have different effects, both on different organisms and on different biological processes within the same organism. The time of exposure (long-term, chronic, repeated) as well as the time of life (early in development, in adulthood) also influence the kind or nature or extent of the biological effects. It is not surprising, then, that regulatory agencies focus on overt, direct toxicity or lethality of the active ingredient of a mixture. It is also not surprising that the focus is on major aspects like carcinogenicity or acute toxicity in humans and animals likely to encounter high concentrations of the compounds being tested for use.

Because so many pesticides have unintended effects and long-term consequences, it makes sense to develop assays of nonlethal effects on reproduction, behavior and nervous system function of exposure to field-levels of the compounds. A rational approach being taken by an increasing number of investigators is to develop behavioral assays in a number of model organisms that have proven to be useful in testing the efficacy of pharmaceutical compounds and that span animal taxa. Understanding the evolutionary similarities among these organisms can provide insight into not only the choice of the model organism, but also the types of behavioral assays that would be most useful. Further, appreciating the differences in mechanism of action or mode of interaction of particular pesticides across evolutionarily distinct taxa will also help inform toxicological testing approaches. While many of the current strategies try to take these kinds of approaches, the continued emphasis on lethality measures, particularly in the licensing approval process, quite often underestimates risks that might lead to longterm morbidity in humans and other animals.

Efforts to explore behavioral effects of pesticides and mixtures of pesticides have been quite successful. As early as 1958, Broley observed abnormal nesting, courtship and reproductive in the Florida bald eagle in areas that were sprayed with DDT [77]. Guppies exposed to the fungicide vinclozolin exhibited changes in body coloration and courtship behavior [78]. The courtship behavior of a number of different bird species, including ringed turtle doves, Japanese quail and Western gulls was adversely affected by exposure to DDT [36]. Many experimental studies in rodents have demonstrated changes in reproductive behavior, locomotory behavior and motivational behavior in response to pesticide exposure [36]. Organophosphates, organochlorines and carbamate pesticides have been shown to alter 
chemosensory behavior in salmon and mice [52, 79], social behavior in fish [80] and mice [81] and behaviors in tadpoles [82], birds [83] and mammals [84].

Behavioral changes can be more sensitive than other measures, like lethality or neurotoxicity. For example, swimming behavior in fish was more sensitive to toxic stress than either lethality or growth [85]. With the growing recognition that behavioral assays can be effective ways to determine possible sublethal and long-term effects of various pesticides, model organisms like the fruit fly Drosophila melanogaster are proving to be effective screens for pesticide sensitivity. Of course, because Drosophila species are insects, they may not be the best models for evaluating effects of pesticides, particularly insecticides, on unintended target organisms. But studies in closely related organisms are crucial for understanding the effects of pesticides on beneficial organisms, like honey bees and other insect pollinators that are also unintended targets of pesticides. Drosophila melanogaster has been historically used for evaluating genotoxicity of pesticides and heavy metals [86]. A number of straightforward behavioral assays, like locomotion, courtship behavior, and aggressive behavior, have proven quite useful in assessing the neurotoxicity and cellular mechanisms of toxicity of various chemicals.

\subsection{Caenorhabditis elegans behavior}

Caenorhabditis elegans (C. elegans), a free living soil nematode with a short life cycle, high fecundity and ease of maintenance in a laboratory, is a valuable model organism to use in neurotoxicology, genotoxicity or behavioral toxicology measures. Its genetic tractability also enables mechanistic studies. The C. elegans genome contains $60-80 \%$ of the genes found in humans and shares many of the same mechanisms of neuron function, development, gene regulation and signal transduction pathways [87]. Early work with this organism examined behavioral toxicity of heavy metals like copper, lead and mercury. Locomotory behaviors like head movement, feeding behavior and simple learning behaviors all showed sensitivity to heavy metal exposure [87- 89]. The herbicide paraquat (methyl viologen) has been extensively studied using C. elegans, particularly in response to emerging epidemiological evidence for an increased incidence of Parkinson's disease in humans exposed to paraquat [90]. Using C. elegans, several potential mechanisms involved in paraquat dopamine neurotoxicity have been described, including oxidative stress leading to reactive oxygen species production [91].

A growing number of studies are accumulating on the adverse behavioral effects on reproduction behaviors, general locomotion or feeding behaviors of sublethal exposure to various pesticides including aldicarb [92], juglone and paraquat [93, 94], organophosphorous compounds $[95,96]$ and carbamates $[97,61,98]$. The LD50 values for these pesticides are similar to those in rats and mice, further validating this model organism for studies of non-target animal toxicity. Sublethal, environmentally relevant concentrations of all of these different environmental contaminants have substantial effects on behavioral processes. Two organophosphorous insecticides, malathion and vapona, inhibit locomotion at sublethal concentrations [99]. Aldicarb, a potent acetylcholinesterase inhibitor, causes paralysis in C. elegans [92], likely because acetylcholine is the excitatory transmitter used at C. elegans neuromuscular junctions and is the neurotransmitter used by one-third of neurons in the C. elegans nervous system [100]. Cholinergic neurons are involved in many behaviors in C. elegans, including 
locomotion, egg laying, feeding and mating. Sublethal concentrations of the organophosphorous insecticide monocrotophos, still in use in developing countries, caused paralysis, reactive oxygen species production and reduced brood size in C. elegans exposed for 4 hours [95]. Chlorpyrifos, a potent acetylcholinesterase inhibitor, also affected brood size after a 72 hour exposure [101]. Dithiocarbamate fungicides like Maneb and Mancozeb, at sublethal concentrations, disrupt dopamine-mediated behaviors [98] and lead to dopamine neurodegeneration [97, 98], which supports epidemiological evidence for a link between carbamate pesticide exposure and Parkinson's disease. Exposure to Mancozeb inhibited locomotion in a dosedependent manner after a 6 hour exposure [98]. In addition, dopamine-specific behaviors like the transition from swimming to crawling were much more vulnerable to Mancozeb than other behaviors mediated by distinct neuronal subtypes [98]. Dopamine-mediated behaviors were disrupted at the lowest concentrations of Mancozeb, whereas behaviors mediated by other neurons, like egg-laying behavior, mediated by serotonergic neurons, were disrupted at slightly higher concentrations of Mancozeb [98]. Importantly, the behavioral deficits are more sensitive to the compounds and the deficits precede overt neurodegeneration, suggesting that behavioral effects of pesticides serve as the earliest biomarker of pesticide toxicity. C. elegans have also been useful for determining gene expression changes due to pesticide exposure [86].

\subsection{Long-term consequences}

Why are behavioral biomarkers desirable when evaluating pesticides for toxicity? First, behavioral responses indicate that the organism is responding to a pesticide. Behavioral changes might well be the first indication of impending toxicity to cells and tissues. Second, some behavioral changes might be reversible, suggesting that eventual damage to the underlying cells/tissues/systems that underlie the behavior can be avoided. Third, behavioral effects, particularly in well-studied organisms, can shed light on mechanistic pathways and systems. Finally, behavioral effects provide insight into population and ecological consequences of long-term exposures.

Persistent changes in reproductive behavior, social behavior or predator avoidance behavior could have long-term consequences for an organism's overall fitness. In one study of the anthropogenic pollution effects on fish communication, researchers examined the turbidity of water on coloration and courtship behavior in Lake Victoria cyclids. Many fish use visual signals to initiate appropriate courtship behavior [102]. The coloration of scales is an important cue for reproductive fitness [102]. In turbid waters, caused by eutrophication and algal blooms or excessive turbulence or increased sediments suspended in the water, these visual communication cues are compromised, which can have an impact on reproduction and possibly overall population characteristics. Over several generations in the turbid water environment, the strong coloration patterns of male cyclids disappeared and the frequency of more mottled, intermediate coloration patterns increased [102]. In another study, endocrine-disrupting properties of a fungicide led to a reduction in the orange coloration spots of male guppies, which in turn reduced the mating success of those males [78]. Male stickleback fish exposed to pesticides showed reduced male nest building and aggressiveness [103], which compromised their reproductive success. Fish grown in waters polluted with mixtures of pesticides and other chemicals exhibited changes in aggressive behavior and predator avoidance escape 
behavior across generations, indicating heritable genetic or epigenetic mechanisms that could have very long-lasting consequences.

Behavioral changes can be due to sensory system dysfunction, to physiological responses or to developmental effects. These changes can differ depending on when an organism is exposed to the chemicals. Chlorpyrifos exposure, at low doses such as measured in the environment, during development of the nervous system, can profoundly affect behavior and susceptibility to disease later in life. Chlorpyrifos at levels below those required to inhibit the intended target acetylcholinesterase, affect the neuroendocrine regulation of development of sexually dimorphic behaviors in rodents. When exposed pre-natally to chlorpyrifos, adult female mice were less aggressively protective of their own nest, but exhibited enhanced maternal behavior towards the pups, as well as increased anxiety behavior [45]. Prenatally-exposed males exhibited increased aggressive behaviors and increased locomotory activity. These social behavior differences correlated with developmental differences in key neurotransmitter and neuroendocrine brain regions that persisted throughout the lifespan.

\subsection{Courtship behavior}

Pesticides can have substantial effects on reproductive success in non-target organisms, which could potentially have considerable negative impacts on wildlife. For example, the highly imperiled Buff-breasted sandpiper (Tryngites subruficollis) is a migratory shorebird that stops over in agricultural fields in the Rainwater Basin area of Nebraska [104]. During their spring migration stopover, these birds forage, rest and engage in social and courtship behaviors that could be impaired by exposure to pesticides. Migratory sea birds, including the albatross and petrel, have measurable levels of organochlorine pesticides in fatty tissues [105]. Flying ability was affected in homing pigeons exposed experimentally to environmentally relevant amounts of chlorpyrifos and aldicarb [106]. An experimental study of chronic low-dose exposure to the organophosphorous pesticide fenitrothion, affected several key reproductive behaviors in male three-spined stickleback fish [107]. Fenitrothion, which in low concentrations is a potent anti-androgen, caused a reduction in male nest-building and impairments in the zigzag courtship dance, both behaviors regulated by testosterone. Male spiders (Rabidosa rabida) exposed to agriculturally relevant concentrations of malathion were unable to perform key courtship behaviors effectively, leading to the males being killed by female spiders prior to copulation [108]. From birds to fish to non-target arthropods, increasing evidence suggests that non-target organisms suffer wide-spread behavioral impairments that could adversely affect population dynamics and success, which could broadly affect numerous ecosystems, even those far away from the site of use of the pesticides.

\subsection{Maternal behavior}

Low concentrations of many pesticides, including organophosphorous, organochlorine and carbamate compounds, affect diverse hormone-mediated systems, thus acting like endocrine disrupting chemicals. Even if a compound does not directly interact with steroid hormone receptors, many systems regulated or mediated by steroid hormones can be influenced via pesticide effects on signal transduction pathways, effects on gene expression or effects on metabolic pathways. Animals exposed to low, chronic levels of pesticides, far below the 
threshold for their intended effects, exhibit impairments in reproductive behaviors and other sexually dimorphic behaviors, particularly if animals are exposed to pesticides in utero or early in development. Chlorpyrifos exposure neonatally has been shown to inhibit DNA synthesis, neuronal differentiation and synaptogenesis in rats [109]. Long-term changes in sexually dimorphic behaviors measured in adult animals exposed perinatally to low levels of chlorpyrifos $[110,40]$ were associated with changes in the development of neural systems, including nontarget serotonergic systems [110]. Both males and females are affected, often in different ways. For example, adult female mice exposed to chlorpyrifos in utero had reduced anxiety behavior and reduced aggression, while adult males showed increased anxiety behavior and aggression. Pre-natally exposed adult females also showed changes in maternal behaviors towards their own young, including reduced nest defense, reduced anxiety in the presence of a strange male approaching the nest and increased licking and crouching of the litter [45]. Adult female mice exposed to the pesticide 2,4 D spent less time nursing their litters even while increasing licking of pups. As a result, litter growth was impaired by the overall reduced time spent nursing. Other animals show impairments in maternal behavior as a result of pesticide exposure: birds living in pesticide-contaminated areas showed parental neglect [111]. Impairments in maternal care can have long-term effects on the offspring, leading to trans-generational effects that are both physiological via developmental changes in the offspring and behavioral as a result of impairments in early socialization learning [45].

\section{Acute versus persistent exposures}

An important issue that has received little direct experimental attention is the differences in response to an environmental contaminant when exposed acutely as compared to living an entire lifespan in the presence of the contaminant. When organisms or populations of organisms are exposed to chemicals throughout their lifespan, physiological and genetic adaptations are possible that would reflect different behavioral effects than in organisms newly exposed to a compound. For example, we found that adult nematodes exposed for 6 hours to Mancozeb exhibited profound locomotory impairment, becoming paralyzed or barely moving and curling up tightly. In addition, their bodies appeared swollen. However, with a 24 hour exposure, those surviving worms exhibited normal locomotory behavior [98] and their bodies were not swollen or misshapen. Offspring exposed throughout development behaved normally as adults (Raley-Susman et al, unpublished observations). A similar result was seen in Daphnia magna exposure for two generations to an environmentally relevant mixture of pesticides [112]. Many studies have demonstrated that exposure during development to low levels of pesticides that can act as endocrine disrupters leads to life long changes in neuroendocrine function, reproductive and sexually dimorphic behaviors [36]. There is also increased risk of developing cancer in childhood or adulthood [113].

Several studies have reported persistent behavioral changes, changes that appear to be independent of targeted effects on acetylcholinesterase inhibition, in rodents exposed to low doses of organophosphate pesticides [114] or carbamate fungicides [115]. Repeated exposures to doses of chlorpyrifos that are below threshold for acetylcholinesterase inhibition caused attention deficits and increased impulsive behavior in rats [116]. Further, long-term exposure 
of zebra fish, Danio rerio, to sublethal concentrations of parathion in the water, led to an increase in general motor activity and food consumption [117]. The behavioral changes occurred in the absence of overt neurological impairment [118] in one study of chronic exposure of mice to rotenone.

\section{Mixtures}

Most organisms, including humans, are exposed to a complex mixture of many different pesticides at once, at varying concentrations and for varying lengths of time. Most freshwater sources contain hundreds of measurable human-produced chemicals. Of the sixteen contaminants present at the highest concentrations in Lake Michigan, eleven of them are pesticides, including diazinon, chlorpyrifos, endosulfan, melathion, atrazine, permethrins, dichlorvos, manganese, zinc, imidacloprid and naphthalenes [119]. Given that the Great Lakes provide 20\% of the Earth's freshwater supply, the effects of these contaminants on the organisms that depend on this water are of critical importance to understand. Depending on the concentrations organisms are exposed to, the mechanisms of action can vary from effects on neuromuscular function to effects on other neurotransmitter systems, hormone systems, cell growth and metabolism. In addition, mixtures could act synergistically or additively. For example, a combination of three common fungicides used routinely in the wine industry acted synergistically at low concentrations to cause greater neurotoxicity than the compounds exerted individually [120]. In another study, long-term exposure to low dose concentrations of two different pesticides, chlorpyrifos and the pyrethroid deltamethrin, caused multiple effects in rat brain tissue. Some effects were additive and some were synergistic [121]. Similarly, azole fungicides have been shown to enhance the toxicity of pyrethroid insecticides to aquatic invertebrates both under laboratory and field conditions [122]. Further, atrazine has been shown to enhance the toxicity of organophosphorus pesticides [123]. Much more work is needed to understand the effects of the complex mixtures of different pesticides and other human contaminants on organismal behavior, fitness and health. It is abundantly clear, however, that the extensive use of pesticides is altering the behavior and possible fitness of many organisms across the globe and in all ecosystems. Because of the persistence of these compounds and the long-term consequences of exposure, some of which are trans-generational, we need to acknowledge that life in a world of pesticides is the new normal.

\section{Summary and conclusions}

Behavioral neurotoxicological and behavioral ecotoxicological approaches need to take prominence in evaluating the short and long-term consequences to wildlife and humans of pesticide use. Behavior can reveal much about the systems and processes affected by pesticides, as well as the mechanisms by which pesticides exert those effects. Behavioral change is often the earliest sign of harmful effects of pesticides, and even low doses of pesticides or pesticide mixtures can lead to long-term behavioral change, particularly when exposures occur during developmental sensitive periods. Because we are living in a world with measureable 
mixtures of pesticides and other human-produced chemicals, it is essential that more work be done to understand how animals, including humans, are living and behaving in this new surrounding milieu.

\section{Acknowledgements}

Thanks to Ms. Melanie Susman for the artwork of Figure 1.

\section{Author details}

Kathleen M. Raley-Susman*

Address all correspondence to: kasusman@vassar.edu

Department of Biology, Vassar College, Poughkeepsie, NY, USA

\section{References}

[1] Hazell, P.B.R. 2009 The Asian Green Revolution. In: International Food Policy Research Institute Discussion Paper 00911. P1-40.

[2] Hirsch, N.P. Neuromuscular junction in health and disease. British Journal of Anaesthesia 2007; 99(1) 132-8.

[3] Lindstrom, J. Nicotinic acetylcholine receptors of muscles and nerves: comparison of their structures, functional roles and vulnerability to pathology. Annals of the New York Academy of Science 2003; 998: 41-52.

[4] Massoulie, J., Millard, C.B. Cholinesterase and the basal lamina at vertebrate neuromuscular junctions. Current Opinions in Pharmacology 2009; 9: 316-325.

[5] Brandan, E, Maldonado, M, Garrido, J, Inestrosa, NC. Anchorage of collagen-tailed acetylcholinesterase to the extracellular matrix is mediated by heparin sulfate proteoglycans. Journal of Cell Biology 1985; 101: 985-992.

[6] Massoulie, J. The origin of the molecular diversity and functional anchoring of cholinesterases. NeuroSignals 2002; 11: 130-143.

[7] Belanger, JH. Contrasting tactics in motor control by vertebrates and arthropods. Integrative and Comparative Biology 2005; 45:672-678. 
[8] Rathmayer, W, Erxleben, C. Identified muscle fibers in a crab. I. Characteristics of excitatory and inhibitory neuromuscular transmission. Journal of Comparative Physiology 1983; 152:411-420.

[9] Cook, C. Earth Island Journal, 2005. http://www.thirdworldtraveler.com/Environment/Spraying-America.html (accessed 20 August 2013).

[10] Casida, JE, Nomura, DK, Vose, SC, Fujioka, K. Organophosphate-sensitive lipases modulate brain lysophospholipids, ether lipids and endocannabinoids. Chemico-Biological Interactions 2008; 175: 355-364.

[11] Pope, CN. Organophosphorus pesticides: Do they all have the same mechanism of toxicity? Journal of Toxicological and Environmental Health B Critical Reviews 1999; 2: $161-181$.

[12] Haynes, KF. Sublethal effects of neurotoxic insecticides on insect behavior. Annual Review of Entomology 1988; 33:149-168.

[13] Source.epa.gov/pesticides/ecosystem/ecorisk.htm

[14] Dominguez, I, Agra, AR, Monaghan, K, Soares, AMVM, Noguiera, AJA. Cholinesterase and glutathione-s-transferease activities in freshwater invertebrates as biomarkers to assess pesticide contamination. Environmental Toxicology and Chemistry 2010; 29(1) 5-18.

[15] Ruark, CD, Hack, CE, Robinson, PJ, Gearhart, JM. Quantitative structure-activity relationships for organophosphates binding to trypsin and chymotrypsis. Journal of Toxicology and Environmental Health A: Current Issues 2011; 74(1) 1-23.

[16] Woodruff, TJ, Zota, AR, Schwartz, JM. Environmental chemicals in pregnant women in the United States: NHANES 2003-4. Environmental Health Perspectives 2011; 119:878-885.

[17] Hertz-Picciotto, I, Delwiche, L. The rise in autism and the role of age at diagnosis. Epidemiology 2009; 20(1) 84-90.

[18] Roberts, EM, English, PB, Grether, JK, Windham, GC, Somberg, L, Wolff, C. Maternal residence near agricultural pesticide applications and autism spectrum disorders among children in the California Central Valley. Environmental Health Perspectives. 2007; 115: 1482-1489.

[19] Eskenazi, B, Marks, AR, Bradman, A, Harley, K, Barr, DB, Johnson, C, et al. Organophosphate pesticide exposure and neurodevelopment in young Mexican-American children. Environmental Health Perspectives 2007; 115: 792-798.

[20] Shelton, JF, Hertz-Picciotto, I, Pessah, IN. Tipping the balance of autism risk: Potential mechanisms linking pesticides and autism. Environmental Health Perspectives 2012; 120(7) 944-951. 
[21] Levin, ED, Addy, N, Nakajima, A, Christopher, NC, Seidler, FJ, Slotkin, TA. Persistent behavioral consequences of neonatal chlorpyrifos exposure in rats. Developmental Brain Research 2001; 130(1) 83-89.

[22] Aluigi, MG, Angelini, C, Falugi, C, Fossa, R, Genever, P, Gallus, L. Interaction between organophosphate compounds and cholinergic functions during development. Chemical and Biological Interactions 2005; 157-8: 305-316.

[23] Yang, D, Lauridsen, H, Buels, K, Chi, LH, La Du, J, Bruun, DA. Chlorpyrifos-oxon disrupts zebra fish axonal growth and motor behavior. Toxicological Sciences 2011; 121(1) 146-159.

[24] Read, DJ, Li, Y, Chao, MV, Cavanagh, JB, Glynn, P. Organophosphates induce distal axonal damage, but not brain oedema, by inactivating neuropathy target esterase. Toxicology and Applied Pharmacology 2010; 245: 108-115.

[25] Crow, JA, Bittles, V, Herring, KL, Borazjani, A, Potter, PM, Ross, MK. Inhibition of recombinant human carboxylesterase 1 and 2 and monoacylglycerol lipase by chlorpyrifos oxon, paraoxon and methyl paraoxon. Toxicology and Applied Pharmacology 2012; 258: 145-50.

[26] Galloway, T, Handy, R. Immunotoxicity of organophosphorous pesticides. Ecotoxicology 2003; 12:345-363.

[27] Stepanovic, RM, Jokanovic, M, Maksimovic, M. Toxicological importance of lymphocyte neuropathy target esterase. Archives in Pharmacology. 1998; 358(2) 5413-5418.

[28] Casale, GP, Cohen, SD, Dicapua, RA. The effects of organophosphate-induced stimulation on the antibody response to sheep erythrocytes in inbred mice. Toxicology and Applied Pharmacology. 1983; 68: 198-205.

[29] Rodgers, KE, Ellefson, DD. Modulation of respiratory burst activity and mitogen response of human peripheral blood mononuclear cells and murine splenocytes and peritoneal cells by malathion. Fundamentals of Applied Toxicology 1990; 14: 309-17.

[30] Rodgers, KE, Ellefson, DD. Mechanism of modulation of murine peritoneal cell function and mast cell degranulation by low doses of malathion. Agents Actions 1992; 35:57.

[31] Eason, CT, Svendsen, C, O'Halloran, K, Weeks, JM. An assessment of the lysosomal neutral red retention test and immune function assay in earthworms (Eisinia andrei) following exposure to chlorpyrifos, benzo-a-pyrene and contaminated soil. Pedobiologia 1999; 43:641-5.

[32] Booth, LH, O'Halloran, K. A comparison of biomarker responses in the earthworm Aporrectodea caliginosa to the organophorphorous insecticides diazinon and chlorpyrifos. Environmental Toxicological Chemistry. 2001; 20: 2494-502. 
[33] Beaman, JR, Finch, R, Gardner, H, Hoffmann, F, Rosencrance, A, Zelikoff, JT. Mammalian immunoassays for predicting the toxicity of malathion in a laboratory fish model. Journal of Toxicology and Environmental Health A 1999; 56: 523-42.

[34] Khalaf-Allah, SS. Effect of water pollution on some haematological, biochemical and immunological parameters in Tilapia nilotica fish. Deutche Tierarztliche Wochenschrift 1999; 106: 67-71.

[35] Crumpton, TL, Seidler, FJ, Slotkin, TA. Developmental neurotoxicity of chlorpyrifos: In vivo and in vitro effects on nuclear transcription factors involved in cell replication and differentiation. Brain Research 2000; 857: 87-98.

[36] Zala, SM, Penn, DJ. Abnormal behaviours induced by chemical pollution: a review of the evidence and new challenges. Animal Behaviour 2004; 68: 649-664.

[37] Levin, ED, Addy, N, Baruah, A, Elias, A, Christopher, N, Seidler, FJ, Slotkin, TA. Prenatal chlorpyrifos exposure in rats causes persistent behavioral alterations. Neurotoxicology and Teratology. 2002; 24:733-741.

[38] Slotkin, TA, Cousins, ML, Tate, CA, Seidler, FJ. Persistent cholinergic presynaptic deficits after neonatal chlorpyrifos exposure. Brain Research 2001; 902: 229-243.

[39] Slotkin, TA, Tate, CA, Cousins, MM, Seidler, FJ. Functional alterations in CNS catecholamine systems in adolescence and adulthood after neonatal chlorpyrifos exposure. Developmental Brain Research. 2002; 287: 163-173.

[40] Icenogle, LM, Christopher, NC, Blackwelder, WP, Caldwell, DP, Qiao, D, Seidler, FJ, Slotkin, TA, Levin, ED. Behavioral alterations in adolescent and adult rats caused by a brief subtoxic exposure to chlorpyrifos during neurulation. Neurotoxicology and Teratology. 2004; 26: 95-101.

[41] Frye, CA, Bo, E, Calamandrei, G, Calza, L, Dessi-Fulgheri, F, Fernandez, M, Fusani, L, Kah, O, Kajta, M, Le Page, Y, Patisaul, HB, Venerosi, A, Wojtowicz, AK, Panzica, GC. Endocrine disrupters: A review of some sources, effects and mechanisms of actions on behavior and neuroendocrine systems. Journal of Neuroendocrinology. 2011; 24:144-159.

[42] Scholz, NL, Truelove, NK, French, BL, Berejikian, BA, Quinn, TP, Sacillas, E and Collier, TK. Diazinon disrupts antipredator and homing behaviors in Chinook salmon (Oncorhynchus tsawytscha). Canadian Journal of Fisheries and Aquatic Sciences. 2000; 57: 1911-1918.

[43] Tait, S, Ricceri, L, Venerosi, A, Maranghi, F, Mantovani, A, Calamandrei, G. Longterm effects on hypothalamic neuropeptides after developmental exposure to chlorpyrifos in mice. Environmental Health Perspectives. 2009; 117: 112-116.

[44] Ricceri, L, Markina, N, Valanzano, A, Fortuna, S, Cometa, MF, Maneguz, A, Calamandrei, G. Developmental exposure to chlorpyrifos alters reactivity to environmen- 
tal and social cues in adolescent mice. Toxicology and Applied Pharmacology. 2003; 191: 189-201.

[45] Venerosi, A, Ricceri, L, Tait, S, Calamandrei, G. Sex-dimorphic behaviors as markers of neuroendocrine disruption by environmental chemicals: The case of chlorpyrifos. Neurotoxicology. 2012; 33: 1420-1426.

[46] Norstrom,RJ, Belikov, SE, Born, EW, Garner, GW, Malone, B, Olpinski, S, Ramsay, MA, Schliebe, S, Stirling, I, Stishov, MS, Taylor MK, Wiig, O. Chlorinated hydrocarbon contaminants in polar bears from eastern Russia, North America, Greenland, and Svalbard: Biomonitoring of Arctic pollution. Archives of Environmental Contaminants and Toxicology. 1998; 35:354-367.

[47] Silva, MH, Gammon, D. An assessment of the developmental, reproductive, and neurotoxicity of endosulfan. Birth Defects Research (Part B). 2009; 86: 1-28.

[48] Davies, TGE, Field, LM, Usherwood, PNR, Williamson, MS. DDT, Pyrethrins, Pyrethroids and insect sodium channels. IUBMB Life 2007; 59(3) 151-162.

[49] Sunderam, RIM, Thomson, GB, Cheng, DMH. Toxicity of endosulfan to native and introduced fish in Australia. Environmental and Toxicological Chemistry 1992; 11(10) 1469-1476.

[50] Jia, H, Liu, L, Sun, Y, Cai, D, Hu, J, Ren, N, Li, Y. Endosulfan in the Chinese environment: monitoring and modeling. Frontiers in Environmental Science Engineering 2012; 6(1) 32-44.

[51] Park, D, Hempleman, SC, Propper, CR. Endosulfan exposure disrupts pheromonal systems in the red-spotted newt: a mechanism for subtle effects of environmental chemicals. Environmental Health Perspectives 2001; 109: 669-673.

[52] Vom Saal, FS, Nagel, SC, Palanza, P, Boechler, M, Parmigiani, S, Welshons, WV. Estrogenic pesticides: binding relative to estradiol in MCF-7 cells and effects of exposure during fetal life on subsequent territorial behavior in male mice. Toxicological Letters. 1995; 77: 343-350.

[53] Palanza, P, Morellini, F, Parmigiani, S, vom Saal, FS. Ethological methods to study the effects of maternal exposure to estrogenic endocrine disrupters: a study with methoxychlor. Neurotoxicology and Teratology 2002; 24:55-69.

[54] Berrill, M, Coulson, D, McGillivray, L, Pauli, B. Toxicity of endosulfan to aquatic stages of anuran amphibians. Environmental and Toxicological Chemistry. 1998; 17: 1738-1744.

[55] Morais, S, Dias, E, Pereira, M. Carbamates: Human exposure and health effects. In: The Impact of Pesticides. AcademyPublish.org. 2012; p. 21-38. 
[56] Weichenthal, S, Moase, C, Chan, P. A review of pesticide exposure and cancer incidence in the Agricultural Health Study cohort. Environmental Health Perspectives. 2010; 118: 1117-1125.

[57] Frazier, LM. Reproductive disorders associated with pesticide exposure. Journal of Agromedicine 2007; 12(1) 27-37.

[58] Meeker, JD, Singh, NP, Ryan, L, Duty, SM, Barr, DB, Herrick, RF, Bennett, DH, Hauser, R. Urinary levels of insecticide metabolites and DNA damage in human sperm. Human Reproduction. 2004; 19(11) 2573-2580.

[59] Wang, A, Costello, S, Cockburn, M, Zhang, X, Bronstein, J, Ritz, B. Parkinson's disease risk from ambient exposure to pesticides. European Journal of Epidemiology. 2011; 26: 547-55.

[60] Easton, A, Guven, K, dePomerai, DI. Toxicity of the dithiocarbamate fungicide Mancozeb to the nontarget soil nematode, Caenorhabditis elegans. Journal of Biochemical and Molecular Toxicology. 2001; 15(1) 15-25.

[61] Benedetto,A, Au,C, Avila,DS, Milatovic,D, Aschner,M. Extracellular dopamine potentiates $\mathrm{Mn}$-induced oxidative stress, lifespan reduction, and dopaminergic neurodegeneration in a BLI-3-dependent manner in Caenorhabditis elegans. PLOSGenetics 2010; 6:1-17.

[62] Freire, C, Koifman, S. Pesticide exposure and Parkinson's disease: Epidemiological evidence of association. NeuroToxicology. 2012; 33: 947-971.

[63] Steinrucken, HC, Amrhein, N. The herbicide glyphosate is a potent inhibitor of 5enol-pyruvylshikimic acid-3-phosphate synthase. Biochemical and Biophysical Research Communications 1980; 94(4) 1207-12.

[64] Giesy, JP, Dobson, S, Solomon, KR. Ecotoxicological risk assessment of Roundup herbicide. IN: Reviews of Environmental Contamination and Toxicology 2000; 167: 35-120.

[65] Walsh, LP, McCormick, C, Martin, C, Stocco, DM. RoundUp inhibits steroidogenesis by disrupting a steroidogenic acute regulatory (StAR) protein expression. Environmental Health Perspectives 2000; 108(8) 769-76.

[66] Romano, RM, Romano, M, Bernardi, MM, Furtado, PV, Oliveira, CA. Prepubertal exposure to commercial formulations of the herbicide glyphosate alters testosterone levels and testicular morphology. Archives of Toxicology 2009; 84(4) 309-17.

[67] Lammer, EJ, Chen, DT, Hoar, RM, Agnish, ND, Benke, PJ, Braun, JT, Curry, CJ, Femhoff, PM. Retinoic acid embryopathy. New England Journal of Medicine. 1985; 313(4) 837-41. 
[68] Paganelli, A, Gnazzo, V, Acosta, H, Lopez, SL, Carrasco, AE. Glyphosate-based herbicides produce teratogenic effects on vertebrates by impairing retinoic acid signaling. Chemical Research in Toxicology. 2010; 23(10) 1586-9.

[69] Richard, S, Moslemi, S, Sipahutar, H, Benachour, W, Seralini, G. Differential effects of glyphosate and RoundUp on human placental cells and aromatase. Environmental Health Perspectives. 2005; 113(6) 716-20.

[70] Kollmeyer, WD, Flattum, RF, Foster, JP, Powell, JE, Schroeder, ME, Soloway, SB. Discovery of the nitromethylene heterocycle insecticides. In: Yamamoto, I, Casida,J (ed) Nicotinoid insecticides and the nicotinic acetylcholine receptor. Tokyo. Springer-Verlag. P71-89.

[71] Tomizawa, M, Latili, B, Casida, JE. Structure and function of insect nicotinic acetylcholine receptors studied with nicotinic insecticide affinity probes. In: Yamamoto, I, Casida,J (ed) Nicotinoid Insecticides and the Nicotinic Acetylcholine Receptor. Tokyo. Springer-Verlag. p. 271-92.

[72] Whitehorn, PR, O'Connor, S, Wackers, FL, Goulson, D. Neonicotinoid pesticide reduces bumble bee colony growth and queen production. Science 2012; 336: 351-2.

[73] Williamson, SM, Wright, GA. Exposure to multiple cholinergic pesticides impairs olfactory learning and memory in honeybees. Journal of Experimental Biology. 2013; 216: 1799-1807.

[74] Nyman, A, Hintermeister, A, Schirmer, K, Aschauer, R. The insecticide imidacloprid causes mortality of the freshwater amphipod Gammarus pulex by interfering with feeding behavior. PLOS One 8: e62472. Doi: 10.1371/journal.pone.0062472.

[75] Capowiez, Y, Berard, A. Assessment of the effects of imidacloprid on the behavior of two earthworm species (Aporrectodea nocturna and Allolobophora icterica) using 2D terraria. Ecotoxicological and Environmental Safety 2006; 64: 198-206.

[76] Tisler, T, Jemec, A, Mozetic,B, Trebse, P. Hazard identification of imidacloprid to aquatic environment. Chemosphere 2008; 76: 907-14.

[77] Broley, C. The plight of the American bald eagle. Audobon Magazine 1958; 60: 162-3, 171.

[78] Baatrup, E, Junge, M, Gunier, RB, Harnly, ME, Reynolds, P, Hertz, A, Von Behren, J. Antiandrogenic pesticides disrupt sexual characteristics in the adult male guppy Poecilia reticulates. Environmental Health Perspectives. 2001; 109: 1063-1070.

[79] Tierney, KB, Sampson, JL, Ross, PS, Sekela, MA, Kennedy, CJ. Salmon olfaction is impaired by an environmentally realistic pesticide mixture. Environmental Science Technology. 2008; 42: 4996-5001. 
[80] Bell, AM. Effects of an endocrine disrupter on courtship and aggressive behavior of male three-spined stickleback, Gasterosteus aculeatus. Animal Behaviour. 2001; 62: 775-780.

[81] Porter, WP, Jaeger, JW, Carlson, IH. Endocrine, immune, and behavioural effects of aldicarb (carbamate), atrazine (triazine) and nitrate (fertilizer) mixtures at groundwater concentrations. Toxicology and Individual Health 1999; 15: 133-150.

[82] Relyea, RA. New effects of Roundup on amphibians: Predators reduce herbicide mortality; herbicides induce antipredator morphology. Ecological Applications 2012; 22(2) 634-647.

[83] Holmes, SB, Boag, PT. Effects of the organophosphorus pesticide fenitrothion on behavior and reproduction in zebra finches. Environmental Research 1990; 53: 62-75.

[84] Flynn, KM, Delclos, KB, Newbold, RR, Ferguson, SA. Behavioural responses of rats exposed to long-term dietary vinclozolin. Journal of Agricultural Food Chemistry. 2001; 49: 1658-1665.

[85] Little, EE, Finger, SE. Swimming behavior as an indicator of sublethal toxicity in fish. Environmental and Toxicological Chemistry. 1990; 9:13-19.

[86] Rand, MD. The growing potential of Drosophila in neurotoxicology. Neurotoxicology and Teratology. 2010; 32: 74-83.

[87] Leung, MCK, Williams, PL, Benedetto, A, Au, C, Helmcke, KJ, Aschner, M, Meyer, JN. Caenorhabditis elegans: An emerging model in biomedical and environmental toxicology. Toxicological Sciences 2008; 106(1) 5-28.

[88] Anderson, GL, Cole, RD, Williams, PL. Assessing behavioral toxicity with Caenorhabditis elegans. Environmental and Toxicological Chemistry. 2004; 23:1235-1240.

[89] Boyd, WA, Cole, RD, Anderson, GL, Williams, PL. The effects of metals and food availability on the behavior of Caenorhabditis elegans. Environmental and Toxicological Chemistry. 2003; 22: 3049-3055.

[90] Liou, HH, Tsai, MC, Chen, CJ, Jeng, JS, Chang, YC, Chen, SY, Chen, RC. Environmental risk factors and Parkinson's disease: A case-control study in Taiwan. Neurology 1997; 48: 1583-1588.

[91] Kim, Y, Sun, H. Functional genomic approach to identify novel genes involved in the regulation of oxidative stress resistance and animal lifespan. Aging 2007; 6:489-503.

[92] Nonet, ML, Saifee, O, Zhao, H, Rand, JB, Wei, L. Synaptic transmission deficits in Caenorhabditis elegans synaptobrevin mutants. Journal of Neuroscience. 1998; 18(1) 70-80.

[93] Wu, Z, Smith, JV, Paramashivarn, V, Butko, P, Khan, I, Cypser, JR, Luo, Y. Ginko biloba extract EGb 761 increases and extends life span of Caenorhabditis elegans. Cell and Molecular Biology 2002; 48(6) 725-731. 
[94] Hartman, P, Childress, E, Beyer, T. Nematode development is inhibited by methyl viologen and high oxygen concentration at a rate inversely proportional to lifespan. J. Gerontol A Biol Sci Med Sci 1995; 50: B322-B326.

[95] Leelaja, BC, Rajini, PS. Biochemical and physiological responses in Caenorhabditis elegans exposed to sublethal concentrations of the organophosphorus insecticide, monocrotophos. Ecotoxicology and Environmental Safety 2013; 94: 8-13.

[96] Roh, JY, Choi, J. Ecotoxicological evaluation of chlorpyrifos exposure on the nematode Caenorhabditis elegans. Ecotoxicology and Environmental Safety 2008; 71: 483-489.

[97] Negga, R, Stuart, JA, Machen, ML, Salva, J, Sizek, AJ, Rishardson, SJ. Exposure to glyphosate-and/or $\mathrm{Mn} / \mathrm{Zn}$-ethylene-bis-dithiocarbamate-containing pesticides leads to degeneration of g-aminobutyric acid and dopamine neurons in Caenorhabditis elegans. Neurotoxicology Research. 2012; 21: 281-290.

[98] Brody, AH, Chou, E, Gray, JM, Pokrywka, NJ, Raley-Susman, KM. Mancozeb-induced behavioral deficits precede structural neuronal degeneration. NeuroToxicology. 2013; 34:74-81.

[99] Williams, PL, Dusenbery, DB. A promising indicator of neurobehavioral toxicity using the nematode Caenorhabditis elegans and computer tracking. Toxicology and Individual Health. 1990; 6: 425-440.

[100] Rand, JB. Acetylcholine. Wormbook 2007 (http://www.wormbook.org/chapters/ www_acetylcholine/acetylcholine.html)

[101] Ruan, QL, Ju, JJ, Li, YH, Liu, R, Pu, YP, Yin, LH, Wang, DY. Evaluation of pesticide toxicities with differing mechanisms using Caenorhabditis elegans. Journal of Toxicology and Environmental Health Part A 2009; 72: 746-751.

[102] Van der Sluijs, I, Gray, SM, Amorim, MCP, Barber, I, Candolin, U, Hendry, AP, Krahe, R, Maan, ME, Utne-Palm, AC, Wagner, HJ, Wong, BBM. Communication in troubled waters: responses of fish communication systems to changing environments. Evolutionary Ecology 2011; 25: 623-640.

[103] Breckels, RD, Neff, BD. Pollution-induced behavioural effects in the brown bullhead (Ameiurus nebulosus). Ecotoxicology 2010; 19:1337-1346.

[104] McCarty, JP, Jorgensen, JG, Wolfenbarger, LL. Behavior of buff-breasted sandpipers (Tryngites subruficollis) during migratory stopover in agricultural fields. PLOS One 2009; 4(11) e8000. doi:10.1371/journal.pone.0008000

[105] Colabuono, FI, Taniguchi, S, Montone, RC. Organochlorine contaminants in albatrosses and petrels during migration in South Atlantic Ocean. Chemosphere 2012; 86: 701-8. 
[106] Moye, JK, Pritsos, CA Effects of chlorpyrifos and aldicarb on flight activity and related cholinesterase inhibition in homing pigeons, Columba livia: Potential for migration effects. Bulletin of Environmental Contamination and Toxicology 2010; 84:677-681.

[107] Sebire, M, Scott, AP, Tyler, CR, Cresswell, J, Hodgson, DJ, Morris, S, Sanders, MB, Stebbing PD, Katsiadaki, I. The organophosphorus pesticide, fenitrothion, acts as an anti-androgen and alters reproductive behavior of the male three-spined stickleback, Gasterosteus aculeatus. Ecotoxicology 2009; 18: 122-133.

[108] Tietzen, WF. Pesticides affect the mating behavior of Rabidosa rabida (Araneae, Lycosidae). Journal of Arachnology 2006; 34: 285-288.

[109] Crumpton, TL, Seidler, FJ, Slotkin, TA. Developmental neurotoxicity of chlorpyrifos in vivo and in vitro: effects on nuclear transcription factors involved in cell replication and differentiation. Brain Research 2000; 857: 87-98.

[110] Aldridge, JE, Livin, Ed, Seidler, FJ, Slotkin TA. Developmental exposure of rats to chlorpyrifos leads to behavioral alterations in adulthood, involving serotonergic mechanisms and resembling animal models of depression. Environmental Health Perspectives 2005; 113: 527-531.

[111] Clotfelter, ED, Bell, AM, Levering, KR. The role of animal behavior in the study of endocrine-disrupting chemicals. Animal Behaviour 2004; 68: 665-676.

[112] Brausch, JM, Salice, CJ. Effects of an environmentally realistic pesticide mixture on Daphnia magna exposed for two generations. Archives of Environmental Contamination and Toxicology 2011; 61: 272-279.

[113] Alavanja, MCR. Pesticides use and exposure extensive worldwide. Reviews in Environmental Health 2009; 24(4) 303-309.

[114] Timofeeva, OA, Sanders, D, Seemann, K, Yang, L, Hermanson, D, Regenbogen, S, Agoos, S, Kallepalli, A, Rastogi, A, Braddy, D, Wells, C, Perraut, C, Seidler, FJ, Slotkin, TA, Levin, ED. Persistent behavioral alterations in rats neonatally exposed to low doses of the organophosphate pesticide, parathion. Brain Research Bulletin 2008; 77: 404-411.

[115] Jia, Z, Misra, HP. Developmental exposure to pesticides zineb and/or endosulfan renders the nigrostriatal dopamine system more susceptible to these environmental chemicals later in life. NeuroToxicology 2007; 28: 727-735.

[116] Middlemore-Risher, ML, Buccafuscu, JJ, Terry, AV. Repeated exposure to low level chlorpyrifos results in impairments in sustained attention and increased impulsivity in rats. Neurotoxicology and Teratology. 2010; 32: 415-424.

[117] Roe, X, Keijzers, R, van Gestel, CAM. Acetylcholinesterase inhibition and increased food consumption rate in the zebra fish, Danio rerio, after chronic exposure to parathion. Aquatic Toxicology. 2003; 64: 457-460. 
[118] Richter, F, Hamann, M, Richter, A. Chronic rotenone treatment induces behavioral effects but no pathological signs of Parkinsonism in mice. Journal of Neuroscience Research 2007; 85: 681-691.

[119] Illinois Environmental Protection Agency. Lake Michigan Monitoring Program. http://www.epa.state.il.us/water/surface-water/lake-michigan-mon.html (accessed 23 August 2013)

[120] Coleman, MD, O’Neil, JD, Woehrling, EK, Ndunge, OBA, Hill, EJ, Menache, A, Reiss, CJ. A preliminary investigation into the impact of a pesticide combination on human neuronal and glial cell lines in vitro. PLOS One 2012; 7(8) c42768. Doi:10.1371/journal.pone.0042768.

[121] Tuzmen, MN, Candan, N, Kaya, E. The evaluation of altered antioxidative defense mechanism and acetylcholinesterase activity in rat brain exposed to chlorpyrifos, deltamethrin, and their combination. Toxicology Mechanisms and Methods 2007; 17: $535-40$

[122] Bjergager, MBA, Hanson,ML, Solomon, KR, Cedergreen, N. Synergy between prochloraz and esfenvalerate in Daphnia magna from acute and subchronic exposures in the laboratory and microcosms. Aquatic Toxicology. 2012; 110-111: 17-24.

[123] Anderson, TD, Lydy, MJ. Increased toxicity to invertebrates associated with a mixture of atrazine and organophosphate insecticides. Environmental Toxicology and Chemistry. 2002; 21: 1507-1514. 